\title{
Cuantificación de carga interna del entrenamiento en jugadores profesionales de fútbol sala
}

\section{Quantification of internal training load in professional players of futsal}

\author{
Ricardo José Ibáñez Pérez*, Guillermo Huerta López y Antonio Juan Meroño
}

Facultad de Ciencias del Deporte, Universidad de Murcia (España).

\begin{abstract}
Resumen: El objetivo era conocer las diferencias existentes de cómo afecta la intensidad del entrenamiento a jugadores profesionales de Fútbol Sala, atendiendo a la posición ocupada en el terreno de juego, y comparar los parámetros de frecuencia cardiaca entre entrenamientos y competición. La investigación se realizó al equipo Plásticos Romero Cartagena F.S., registrando las medias de frecuencia cardiaca máxima con pulsómetros y la recogida de la Percepción Subjetiva del Esfuerzo, a través de la escala de Borg. Los resultados obtenidos no mostraron diferencias significativas respecto a la carga cuantificada en las tres posiciones, además la carga interna se manifestó de forma más exigente en jugadores que ocupaban la posición de "Ala" y "Pívot". Los parámetros de frecuencia cardiaca registrados en entrenamientos se encontraban muy distanciados de los datos de competición recogidos por otros estudios y la percepción subjetiva del esfuerzo se mostró como un método válido para la cuantificación de la carga.

Palabras claves: Fútbol Sala, Posiciones, Frecuencia Cardiaca, Percepción Subjetiva del Esfuerzo, Control, Carga de Entrenamiento, Rendimiento.
\end{abstract}

Abstract: The objective was to know the existing differences in how the intensity of training affects professional futsal players, taking into account the position occupied on the pitch, and compare the heart rate parameters between training and competition. The investigation was carried out to the Romero Cartagena F.S. Plastics team, recording the maximum heart rate means with heart rate monitors and the collection of the Subjective Perception of Effort, through the Borg scale. The results obtained did not show significant differences with respect to the quantified load in the three positions, in addition the internal load manifested itself in a more demanding way in players who occupied the position of "Wing" and "Pivot". The heart rate parameters recorded in training were very distant from the competition data collected by other studies and the subjective perception of effort was shown as a valid method for the quantification of the load.

Keywords: Indoor Football, Positions, Heart Rate, Rate of Perceived Exertion, Quantification, Training Load, Performance.

\section{Introducción}

Según Bortoli, Bortoli y Márquez (2002), conseguir e intentar llegar al alto rendimiento en deportes colectivos origina y promueve una búsqueda constante de medios, herramientas, y procedimientos que posibiliten y mejoren el trabajo deportivo. Por ello, llegar a obtener un alto rendimiento deportivo en la preparación física de los deportes colectivos como por ejemplo en el Fútbol Sala resulta esencial, ya que uno de los elementos que define la aptitud del deportista en el transcurso de la competición es su estado físico (Sanabria y Agudelo, 2011).

Como mencionan Vallés, Fernández-Ozcorta y Fierro (2017) el medio por el que se consigue la condición ideal del deportista es a través del entrenamiento, entendido como el procedimiento en el que el deportista es expuesto a estímulos programados, los cuales inducen una fatiga determinada, que tras una adecuada fase de recuperación proporcionan un aumento del rendimiento. Por tanto, según Campos-Vázquez (2012), uno de los cargos del preparador físico que se debe tener en cuenta por su importancia, es el control del entrenamiento ya que una de las funciones primordiales del preparador físico es definir las diferentes cargas de trabajo que provoquen y tenga un impacto de adaptación en el deportista.

Dirección para correspondencia [Correspondence address]: Ricardo José Ibáñez Pérez. E-mail: ricardojose.ibanez@um.es
Es por ello por lo que en deportes colectivos cuantificar la carga de entrenamiento es una de las cuestiones que más obsesionan a los preparadores físicos (Álvarez, Manonelles y Corona, 2004), tanto su control, como su manejo y registro a la hora de plantear la planificación de una temporada (Cuadrado-Reyes, Chirosa-Ríos, Chirosa-Ríos, Martín-Tamayo y Aguilar-Martínez, 2012).

Teniendo en cuenta que como mencionan Gómez-Díaz, Bradley, Díaz y Pallares (2013), "la carga de entrenamiento es entendida como el conjunto de exigencias mecánicas, biológicas y psicológicas que provocan un estado de desequilibrio en el cuerpo del atleta" (p.656), una estimación rigurosa de la carga es un paso esencial para la planificación y periodización del entrenamiento (Campos-Vázquez, 2012), debido a que su adecuado registro y utilización se ha manifestado como esencial para mejorar el rendimiento en jugadores de fútbol de alto nivel (Gómez-Díaz et al., 2013).

Además, definir de forma ajustada la carga de entrenamiento, sería la forma correcta de conseguir las metas y objetivos marcados por el cuerpo técnico del equipo (CuadradoReyes et al., 2012), así como el procedimiento adecuado para averiguar y percibir la aproximación entre el rendimiento, las adaptaciones esperadas y las conseguidas (Álvarez y Murillo, 2016). 
De este modo, evaluar y determinar la carga de entrenamiento nos aportará información útil para el diseño de entrenamientos más individualizados. Por lo tanto, la cuantificación de la carga debe ser primordial, tratando de conseguir de manera conjunta los objetivos de: individualizar los programas de entrenamiento, analizar las conexiones entre factores de rendimiento del deporte y la cantidad realizada de carga, y eludir situaciones de sobreentrenamiento o desentrenamiento (Campos-Vázquez, 2012).

En deportes colectivos como el fútbol sala, donde hay competición cada siete días, durante un ciclo con una duración próxima a 40 semanas, y con unas características particulares, produce que la panificación de las cargas y periodos de entrenamiento sean dispares y se alejen de las planificaciones o programaciones de los deportes donde las competiciones están mucho más alejadas unas de otras en el tiempo (Álvarez, Manonelles, Giménez y Nuviala, 2009).

Además, la cuantificación de la carga es particularmente difícil de establecerla en deportes colectivos por el impedimento que los elementos tácticos suponen (Campos-Vázquez, 2012). Por eso para controlar y determinar las cargas en deportes colectivos se debe tener en cuenta la discontinuidad de los esfuerzos, lo que nos supondrá una dificultad para determinarlas (Cuadrado y Grimaldi, 2011). Por ello, como mencionan Pascual, Llorca, Carbonell y Pérez-Turpín (2016), "se ha de tener en cuenta su discontinuidad, ya que estamos analizando un deporte acíclico" (p.256).

Dependiendo de la especialidad deportiva, la verificación del entrenamiento se realiza a partir de la carga externa e interna (Vallés et al., 2017). Es por esto, por lo que cada vez se ha generado más curiosidad en los preparadores físicos el momento de verificar y determinar la monitorización de la carga externa e interna, al igual que la fatiga y su proceso. De esta manera se quiere comprender las adaptaciones elaboradas por el sistema de entrenamiento y eliminar secuelas negativas como lesiones y dolencias (Tapia-López, 2017).

Por lo tanto, en deportes colectivos, se puede definir a la carga establecida por el entrenador como carga externa, entretanto se califica como carga interna al estrés fisiológico tolerado por los jugadores (Campos-Vázquez, 2012). La carga externa está formada por marcadores como el tiempo de entreno, ejercicios, cantidad de entrenamientos, etc.

Por otro lado la carga interna está determinada por los parámetros fisiológicos que produce el deportista como respuesta a la carga externa. Algunos de estos parámetros fisiológicos pueden ser la frecuencia cardiaca máxima (FC Máx¹), lactato

1 FC Máx: Frecuencia Cardiaca Máxima. La frecuencia cardíaca máxima es el número de latidos máximo que puede alcanzar tu corazón durante un minuto sometido a esfuerzo.
$\left(\mathrm{LAC}^{2}\right)$ y consumo máximo de oxigeno $\left(\mathrm{VO}_{2} \mathrm{Max}^{3}\right)$ (Vallés et al., 2017).

Habitualmente, la totalidad de métodos de control de la carga se han ajustado y realizado sobre la carga externa, pero hay que tener en cuenta el estrés fisiológico y psicológico establecido al jugador por la carga interna (Tapia-López, 2017).

La carga interna nos proporciona datos sobre los niveles de adaptación y recuperación de la fatiga, de esta forma nos posibilita modificar la carga del entrenamiento siguiente. Además, ante una carga externa semejante dos jugadores pueden expresar cargas internas dispares o desiguales (Tapia-López, 2017).

Por eso, establecer la carga adecuada del entrenamiento para posibilitar que los jugadores continúen obteniendo adaptaciones o lleguen a un estado óptimo de forma se complica cuando trabajamos con un grupo de jugadores diverso y variado con diferentes características (Álvarez et al., 2004).

También hay que destacar que además de las características propias de cada jugador, la posición determinada que desempeñen en la pista, pueden cambiar los resultados en cuanto a la intensidad y duración del esfuerzo producido en el transcurso de la competición o entrenamiento (García-Jiménez y Yuste, 2010).

Por todos los motivos anteriores habrá determinados métodos de entrenamiento que beneficiarán más a unos jugadores que a otros en momentos puntuales de la temporada. Por eso, el preparador físico debe ser cuidadoso y acondicionar las cargas a las características de los jugadores dentro de lo posible (Álvarez et al., 2004).

También es necesario verificar la carga interna del entrenamiento para adecuar la intensidad del entrenamiento y trabajar en niveles cercanos y semejantes a la competición, ya que se estima que la competición es la manera más eficaz de control, debido a que los parámetros del rendimiento forman parte de ella (Barbero, Grande y Soto-Hermoso, 2004).

Ahora existen numerosas formas de cuantificar la carga interna a la que son sometidos los jugadores. Se pueden encontrar métodos como la frecuencia cardiaca (FC), el consumo de oxígeno $\left(\mathrm{VO}_{2}\right)$ o la concentración de ácido láctico (LAC) (Cuadrado-Reyes et al, 2012), pero la mayoría de ellas se cen-

2 LAC: Lactato. El ácido láctico es un compuesto químico. Es una sustancia que genera el organismo que, en principio, es beneficiosa pero el exceso puede generar bajas en el rendimiento e incluso dańos musculares. Se produce principalmente en las células musculares y en los glóbulos rojos cuando descompone carbohidratos en condiciones de niveles bajos de oxígeno. Es decir, es una fuente de energía para el cuerpo humano.

$3 \mathrm{VO}_{2}$ Max: Volumen de oxigeno máximo. es la cantidad máxima de oxígeno $(\mathrm{O} 2)$ que el organismo puede absorber, transportar y consumir en un tiempo determinado, es la sangre que nuestro organismo puede transportar y metabolizar.También se denomina consumo máximo de oxígeno o capacidad aeróbica. 
tran en la frecuencia cardiaca (Tapia-López, 2017). De este modo, numerosos estudios afirman que uno de los mejores parámetros para cuantificar la intensidad y carga interna del entrenamiento es el control de la frecuencia cardiaca (Castañer, Saüch, Camerino, Sánchez-Algarra y Anguera, 2014).

Por otro lado, este control de la carga interna del futbolista a través de marcadores fisiológicos necesita una cantidad inmensa de recursos materiales y humanos que la mayoría de veces no se encuentran disponibles ni en el fútbol profesional (Gómez-Díaz et al., 2013), y de nuevo aparece la misma dificultad y contratiempo, la utilización de estos sistemas de cuantificación de la carga interna necesita de instrumentos costosos para los equipos que no sean profesionales y no puedan permitirse pulsómetros y analizadores de lactato (TapiaLópez, 2017).

Por consiguiente se hace necesario el perfeccionamiento de herramientas alternas que ofrezcan la posibilidad de cuantificar de forma práctica y con valores admisibles la carga interna del jugador como respuesta a los diferentes estímulos establecidos en el entrenamiento. En el fútbol profesional y en la investigación científica el método más usado para cuantificar la carga de forma asequible, útil y provechosa, es la percepción subjetiva del esfuerzo, ya sea en su fórmula original o transformándola en ocasiones posteriores para la que se han establecido registros de un validez superior (Gómez-Díaz et al., 2013).

Por lo tanto, numerosos estudios han destacado el valor y la utilidad de este método para determinar y cuantificar la carga y fatiga de los jugadores en entrenamientos y competición, definido siempre desde dos variantes anexas e inseparables, la física y la psicológica (Gómez-Díaz et al., 2013).

Actualmente ha habido un aumento en la utilización del método $\mathrm{PSE}^{4}$ entre el cuerpo técnico de los equipos para verificar la carga de entrenamiento. El empleo de este método se basa en la oportunidad de que el propio jugador determine su fatiga y manifieste por sí mismo el grado de esfuerzo realizado en el entrenamiento (Tapia-López, 2017).

En los deportes colectivos, la PSE es uno de los indicadores más utilizados para determinar la carga interna en entrenamientos y competiciones. Todo esto es consecuencia de su sencillo uso y de su asequibilidad. Se puede utilizar para cuantificar y determinar la carga de un ejercicio determinado así como la de una sesión de entrenamiento en su totalidad (Cuadrado-Reyes et al., 2012), y a través de un solo número que expresa el esfuerzo y fatiga combinados en los ejercicios que forman el entrenamiento, el jugador o deportista establece la intensidad del entrenamiento (Álvarez, Murillo, Usan, Ros y Manonelles, 2016).

4 PSE: Percepción subjetiva del esfuerzo. Es la intensidad subjetiva de esfuerzo, tensión, malestar y/o fatiga que se experimenta durante el ejercicio físico.
Su utilización no debe estancarse únicamente en establecer las resoluciones que los jugadores hacen sobre el entrenamiento realizado, sino que puede ser efectivo entre los preparadores físicos y entrenadores para confirmar que las planificaciones de entrenamientos se equiparan a las sesiones realizadas (Tapia-López, 2017).

Además como confirman Castañer et al., (2014), “ya existe literatura que ha corroborado la correlación existente entre la FC y la RPE"” (p.84). Es por esto que en deportes colectivos los sistemas de cuantificación como son la FC y la PSE son empleados en competición, ya que se ha comprobado que son métodos apropiados para estimar, intensidad, gasto energético y diferentes respuestas fisiológicas en competición (Calahorro, Torres-Luque y Lara-Sánchez, 2013).

Consecuentemente el objetivo del estudio es la cuantificación de la carga interna de los jugadores de un equipo profesional de Fútbol Sala en función de las distintas posiciones que pueden ocupar ("Cierre", "Ala", "Pívot"), a través de la FC y la PSE, y de esta forma ver si existen diferencias significativas en la forma en que la intensidad del entrenamiento afecta a la carga interna según las diferentes posiciones que pueden ocupar en el terreno de juego los jugadores. Observar si los valores analizados están cercanos a los registrados en competición por otros estudios. Y estudiar si la percepción subjetiva del esfuerzo se muestra como un método válido para la cuantificación de la carga interna en jugadores de Fútbol Sala.

\section{Material y método}

\subsection{Muestra}

Los participantes objeto de estudio que formaron parte en este trabajo de investigación han sido nueve jugadores con una media de 28 ańos de edad, del equipo Plásticos Romero Cartagena de Fútbol Sala, equipo profesional y de alto nivel que milita en la primera división de la Liga Nacional española de Fútbol Sala (LNFS).

Todos ellos fueron informados previamente del estudio y tenían experiencia previa en el uso de pulsómetros, conocían el protocolo a seguir para establecer los valores en la escala de Borg (1990), ya que lo habían realizado en sesiones de entrenamiento anteriores.

\subsection{Material y Procedimiento}

La investigación se realizó en un periodo abarcado desde el seis de marzo hasta el dieciocho de abril de 2018, siendo registradas un total de catorce sesiones de entrenamiento de

5 RPE: Rate of Perceived Exertion. Percepción subjetiva del esfuerzo.

Cuantificación de carga interna del entrenamiento en jugadores profesionales de... SPORT TK, 9(2), 75-86 
una hora y media cada una realizadas en el Palacio de Deportes de Cartagena (Murcia).

El registro de la frecuencia cardíaca se ha realizado mediante seis monitores de ritmo cardíaco "Polar Bluetooth Smart H7". Los datos recogidos de FC eran anotados en una hoja de registro diseñada exclusivamente para la investigación y para su posterior análisis y desarrollo en una hoja Excel. Contando con el consentimiento del Presidente del equipo, así como del cuerpo técnico y de todos los jugadores.

Se anotaron los datos de FC de cada jugador justo al finalizar cada ejercicio de la sesión de entrenamiento y se analizó la carga interna de forma íntegra, ya que como afirman Álvarez et al. (2004), "las situaciones reales de juego en los entrenamiento suelen efectuarse después de un trabajo previo que puede ocupar más de la mitad del entrenamiento, por lo que la carga hay que entenderla desde una perspectiva global y no de forma fraccionada e independiente a lo largo de las diferentes partes de una sesión de entrenamiento" (p.48).

Por lo que respecta al registro de la Percepción Subjetiva del Esfuerzo (PSE) se utilizó la escala de Borg modificada del 1-10, donde uno (1) era "muy muy suave y diez (10) era "extremadamente máximo” Borg, (1990), la cual se aplicó a cada uno de los jugadores al finalizar el entrenamiento, justo antes de la retirada al vestuario.

\subsection{Análisis estadísticos}

La recogida de datos, el tratamiento y el análisis se realizaron utilizando un software de hojas de cálculo de Microsoft Office Excel 2010 y Microsoft Office Word 2010. Los datos se presentan como medias de los valores de frecuencia cardiaca máxima y de percepción subjetiva del esfuerzo recogidos en las catorce sesiones de entrenamiento.

\section{Resultados}

En la Tabla 1, se muestran los datos de las variables de frecuencia cardiaca recogidas en las catorce sesiones de entrenamiento. En los resultados se observa la media obtenida según las distintas posiciones que los jugadores pueden ocupar en el terreno de juego.
Tabla 1. Media de la FC Máx en P/M6, de los jugadores del Plásticos Romero Cartagena Fútbol Sala. 2017-2018.

\begin{tabular}{lccc}
\hline & $\overline{\mathrm{x}}$ & $\overline{\mathrm{x}}$ & $\overline{\mathrm{x}}$ \\
& FC Máx "Cierre" & FC Máx "Ala" & FC Máx "Pívot" \\
\hline Sesión 1 & $132 \mathrm{P} / \mathrm{M}$ & $164 \mathrm{P} / \mathrm{M}$ & $148 \mathrm{P} / \mathrm{M}$ \\
Sesión 2 & $134 \mathrm{P} / \mathrm{M}$ & $152 \mathrm{P} / \mathrm{M}$ & $151 \mathrm{P} / \mathrm{M}$ \\
Sesión 3 & $152 \mathrm{PM}$ & $163 \mathrm{P} / \mathrm{M}$ & $166 \mathrm{P} / \mathrm{M}$ \\
Sesión 4 & $124 \mathrm{P} / \mathrm{M}$ & $155 \mathrm{P} / \mathrm{M}$ & $155 \mathrm{P} / \mathrm{M}$ \\
Sesión 5 & $157 \mathrm{P} / \mathrm{M}$ & $132 \mathrm{P} / \mathrm{M}$ & $138 \mathrm{P} / \mathrm{M}$ \\
Sesión 6 & $114 \mathrm{P} / \mathrm{M}$ & $132 \mathrm{P} / \mathrm{M}$ & $158 \mathrm{P} / \mathrm{M}$ \\
Sesión 7 & $143 \mathrm{P} / \mathrm{M}$ & $146 \mathrm{P} / \mathrm{M}$ & $147 \mathrm{P} / \mathrm{M}$ \\
Sesión 8 & $128 \mathrm{P} / \mathrm{M}$ & $142 \mathrm{P} / \mathrm{M}$ & $124 \mathrm{P} / \mathrm{M}$ \\
Sesión 9 & $117 \mathrm{P} / \mathrm{M}$ & $133 \mathrm{P} / \mathrm{M}$ & $143 \mathrm{P} / \mathrm{M}$ \\
Sesión 10 & $161 \mathrm{P} / \mathrm{M}$ & $141 \mathrm{P} / \mathrm{M}$ & $132 \mathrm{P} / \mathrm{M}$ \\
Sesión 11 & $133 \mathrm{P} / \mathrm{M}$ & $138 \mathrm{P} / \mathrm{M}$ & $155 \mathrm{P} / \mathrm{M}$ \\
Sesión 12 & $130 \mathrm{P} / \mathrm{M}$ & $149 \mathrm{P} / \mathrm{M}$ & $142 \mathrm{P} / \mathrm{M}$ \\
Sesión 13 & $174 \mathrm{P} / \mathrm{M}$ & $156 \mathrm{P} / \mathrm{M}$ & $134 \mathrm{P} / \mathrm{M}$ \\
Sesión 14 & $115 \mathrm{P} / \mathrm{M}$ & $132 \mathrm{P} / \mathrm{M}$ & $127 \mathrm{P} / \mathrm{M}$ \\
\hline
\end{tabular}

En la Tabla 1, observamos que en la investigación realizada sobre los jugadores del Plásticos Romero Cartagena Fútbol Sala en cuanto a la posición de "Ala" se han recogido los valores más altos de la media de FC Máx en seis sesiones de entrenamiento.

Los mismos resultados y valores se han obtenido en la investigación en cuanto a la posición de "Pivot", ya que en otras seis sesiones de entrenamiento su media de FC Máx ha sido la más elevada. Esta evidencia en cuanto a que la media de FC Máx haya sido casi siempre más elevada en la posición de "Ala" y "Pívot" puede estar relacionada con la distancia recorrida en los entrenamientos, ya que puede ser mayor que la realizada por los jugadores en la posición de "Cierre".

En los valores recogidos en la investigación en la posición de "Cierre" observamos que solamente se obtuvieron los valores de la media de FC Máx más altos en tres sesiones, y por el contrario hubo once sesiones donde los valores de la media de FC Máx fueron más bajos. La explicación a este anomalía puede ser que uno de los sujetos que ocupaba la posición de cierre tenía una FC en reposo muy baja, y por tanto esto le hacía tener una FC Máx menor que el resto de los jugadores que ocupaban la posición de "Cierre", por lo que los valores medios en la medición de la posición de "Cierre" eran menores.

Figura 1. Evolución de la media de FC Máx según posiciones ocupadas en el terreno de juego por los jugadores del Plásticos Romero Cartagena Fútbol Sala.

En la Figura 1, se muestra la evolución de las distintas medias de FC Máx en función posiciones de los jugadores

6 P/M: Pulsaciones por minuto del corazón. 
del Plásticos Romero Cartagena de Fútbol Sala durante las catorce sesiones de entrenamientos registradas.

Hay que destacar que las posiciones de "Ala" y "Pivot", es donde se han conseguido el mayor número de sesiones con registros más altos de FC Máx. Sin embargo la media de FC
Máx más elevada fue registrada en la sesión 13 con una media de 175 P/M por la posición de "Cierre". A su vez, la posición de "Cierre" fue la posición que en un menor número de sesiones registró la media de FC Máx más alta.

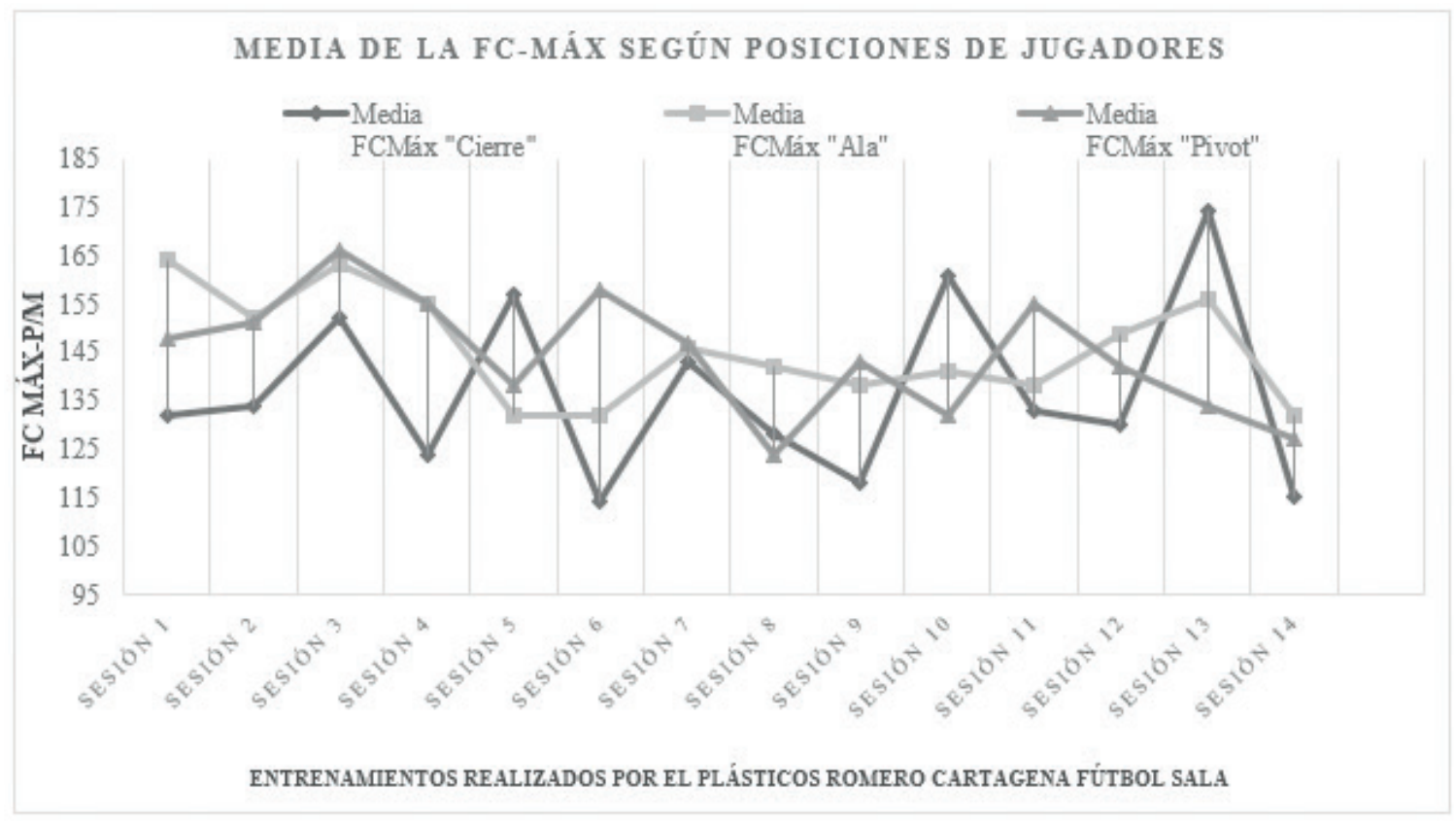

Figura 1. Evolución de la media de FC Máx según posiciones ocupadas en el terreno de juego por los jugadores del Plásticos Romero Cartagena Fútbol Sala.

En la Figura 1, también se observa que la media más elevada de FC Máx respecto al total de las catorce sesiones ha sido conseguida por la posición de "Ala" y por la de posición de "Pívot" con una frecuencia cardiaca de $145 \mathrm{P} / \mathrm{M}$. En último lugar los valores más bajos de la media de FC Máx en el total de las catorce sesiones de entrenamiento pertenecen a la posición de "Cierre" con un valor de frecuencia cardiaca de $139 \mathrm{P} / \mathrm{M}$.

En la Tabla 2, se recogen las variables de percepción subjetiva del esfuerzo recogidas en las catorce sesiones de entrenamiento. En los resultados se muestra la media obtenida de PSE en la escala de Borg según las distintas posiciones que los jugadores pueden ocupar en el terreno de juego.
Tabla 2. Media de la PSE en la escala de Borg (ver imagen 1) según posiciones de los jugadores del Plásticos Romero Cartagena Fútbol Sala. 2017-2018.

\begin{tabular}{lccc}
\hline & $\overline{\bar{x}}$ & $\overline{\mathrm{x}}$ & $\overline{\bar{x}}$ \\
& PSE “Cierre" & PSE “Ala” & PSE "Pívot” \\
\hline Sesión 1 & 4 & 5 & 4 \\
Sesión 2 & 3 & 5 & 6 \\
Sesión 3 & 5 & 6 & 5 \\
Sesión 4 & 4 & 5 & 5 \\
Sesión 5 & 4 & 5 & 4 \\
Sesión 6 & 7 & 6 & 6 \\
Sesión 7 & 6 & 4 & 4 \\
Sesión 8 & 5 & 5 & 4 \\
Sesión 9 & 5 & 6 & 5 \\
Sesión 10 & 6 & 7 & 6 \\
Sesión 11 & 4 & 4 & 6 \\
Sesión 12 & 5 & 6 & 5 \\
Sesión 13 & 6 & 4 & 4 \\
Sesión 14 & 3 & 3 & 4 \\
\hline
\end{tabular}


En la Tabla 2 se observa que las medias de PSE de los jugadores del Plásticos Romero Cartagena Fútbol Sala han sido muy similares en las tres posiciones analizadas en el registro de las catorce sesiones de entrenamiento.

Se puede contemplar que la posición que más número de veces ha establecido unos valores de PSE mayores en los entrenamientos recogidos en la investigación respecto a las otras posiciones ha sido la posición de "Ala", en hasta ocho sesiones de entrenamiento.

También observamos que en dos sesiones realizadas, la media de PSE de la posición de "Ala" ha sido similar a la de la posición de "Pivot", coincidiendo solamente una vez con la posición de "Cierre".

Por otro lado, las medias de PSE más elevadas en el resto de sesiones se han obtenido en la posición de "Pívot" y de "Cierre", ya que se registraron los valores más elevados de la PSE en cuatro sesiones en ambas posiciones.

Figura 2. Evolución de la media de PSE según posiciones ocupadas en el terreno de juego por los jugadores del Plásticos Romero Cartagena Fútbol Sala.

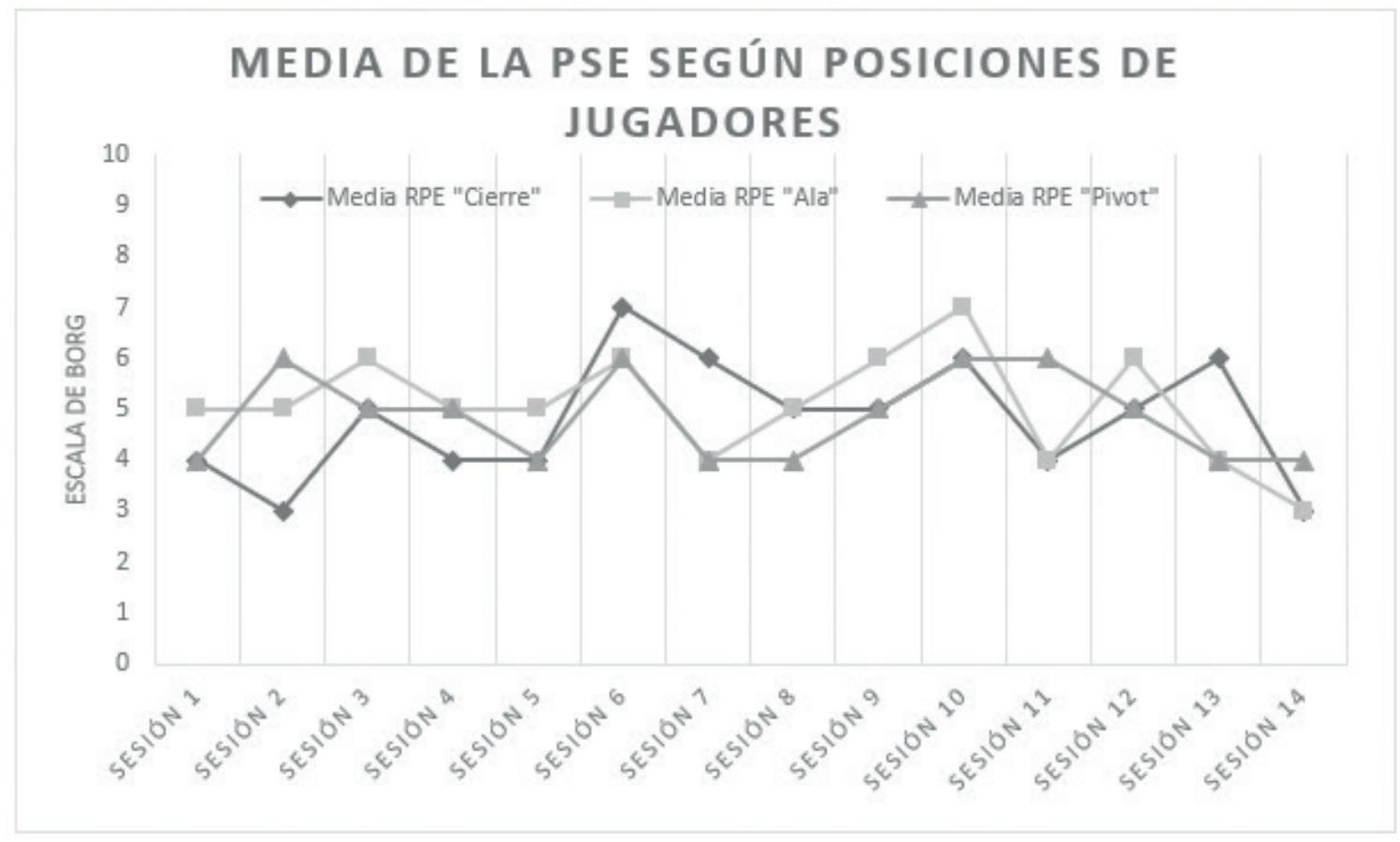

Figura 2. Evolución de la media de PSE según posiciones ocupadas en el terreno de juego por los jugadores del Plásticos Romero Cartagena Fútbol Sala.

En la Figura 2, se muestra la evolución de las distintas medias de PSE en la escala de Borg en función de las posiciones ocupadas por los jugadores del Plásticos Romero Cartagena de Fútbol Sala durante las catorce sesiones de entrenamientos.

Hay que destacar que aunque los valores analizados han sido muy similares en todas las posiciones, la PSE más alta en un mayor número de sesiones ha sido en la posición de "Ala". En la Figura 2, se observa que la media de la PSE obtenida respecto al total de las catorce sesiones de entrenamiento ha sido similar en las tres posiciones ("Ala", "Pívot" y "Cierre") con un valor de cinco (5), que en la escala de Borg utilizada corresponde a una percepción del esfuerzo con la calificación de "Duro".
En la Figura 2, se puede ver una evolución inversa en algunos tramos de la gráfica, y directa entre otros respecto a las variables reflejadas de la media de FC Máx y la media de la PSE en la posición de "Cierre".

Como dato más significativo se puede destacar la sesión 6, en la cual se observa que la media de la FC Máx fue una de las más bajas registradas pero por el contrario la media de la PSE fue la más alta de las catorce sesiones registradas de entrenamiento.

Esta evolución indirecta puede deberse al momento en el que se registraba la PSE en los entrenamientos, ya que era justo antes de marcharse los jugadores a las duchas. 


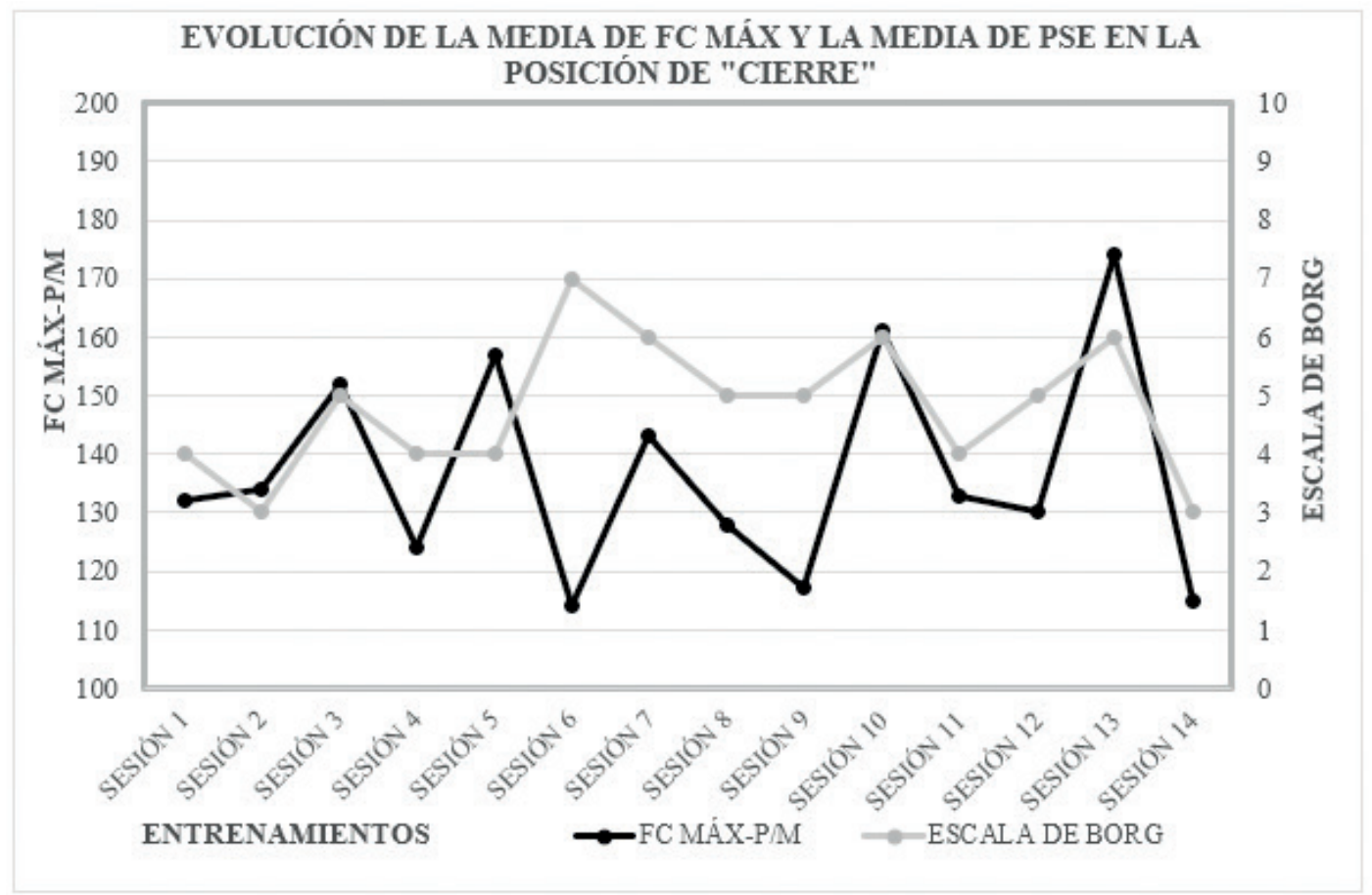

Figura 3. Evolución de la media de FC Máx y la media de PSE en la posición de "Cierre" a los largo de las catorce sesiones de entrenamiento realizadas por los jugadores del Plásticos Romero Cartagena Fútbol Sala.

Esto puede provocar una percepción del esfuerzo distorsionada por los jugadores, debido a que aunque la primera parte del entrenamiento fuera de intensidad leve, si los últimos ejercicios requerían de un gran esfuerzo su PSE se podía ver modificada, es decir, hay una posibilidad de que los jugadores no analizaran la sesión de forma global, y de esta forma su rango en la escala de Borg fuese mayor debido a la última parte del entrenamiento.

En la Figura 4, se puede ver una evolución inversa en algunos tramos de la gráfica, y directa entre otros respecto a las variables reflejadas de la media de FC Máx y la media de la PSE en la posición de "Ala".

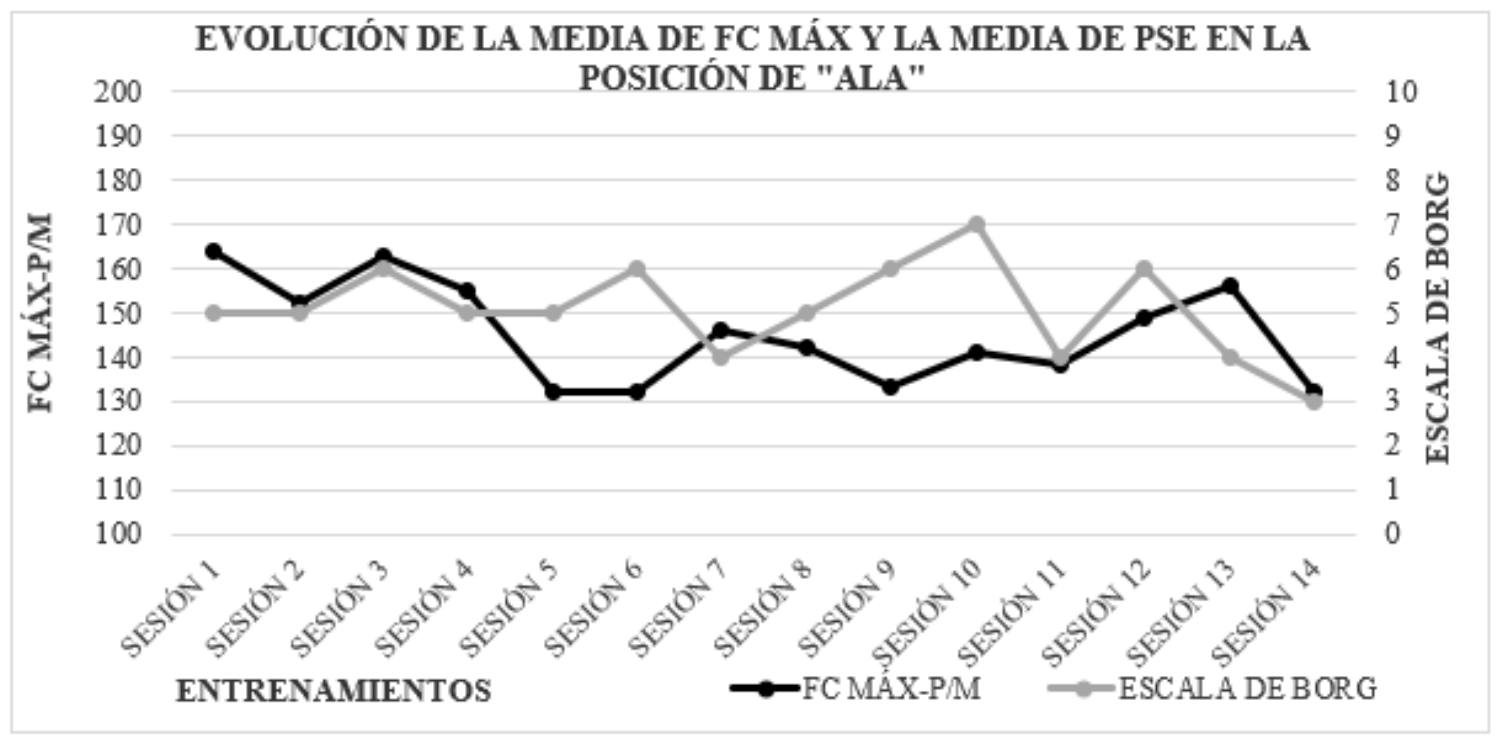

Figura 4. Evolución de la media de FC Máx y la media de PSE en la posición de "Ala" a los largo de las catorce sesiones de entrenamiento realizadas por los jugadores del Plásticos Romero Cartagena Fútbol Sala. 
Se puede destacar la relación indirecta respecto a la evolución de las sesiones 5-6 y 8-9-10, en la cual la media de FC Máx era muy similar, pero la media de la PSE se incrementó llegando a registrarse en la sesión 10 el dato más alto de la PSE en la posición de "Ala".

Esto puede deberse a la carga de entrenamiento de esa se- mana, ya que aunque la media de FC máx era similar, los jugadores iban acumulando fatiga de la sesión previa del día anterior, por lo que su PSE al día siguiente se vio modificada. En cuanto a la evolución de las sesiones 2-3-4 se puede ver una relación directa de la media de FC Máx y la media de la PSE.

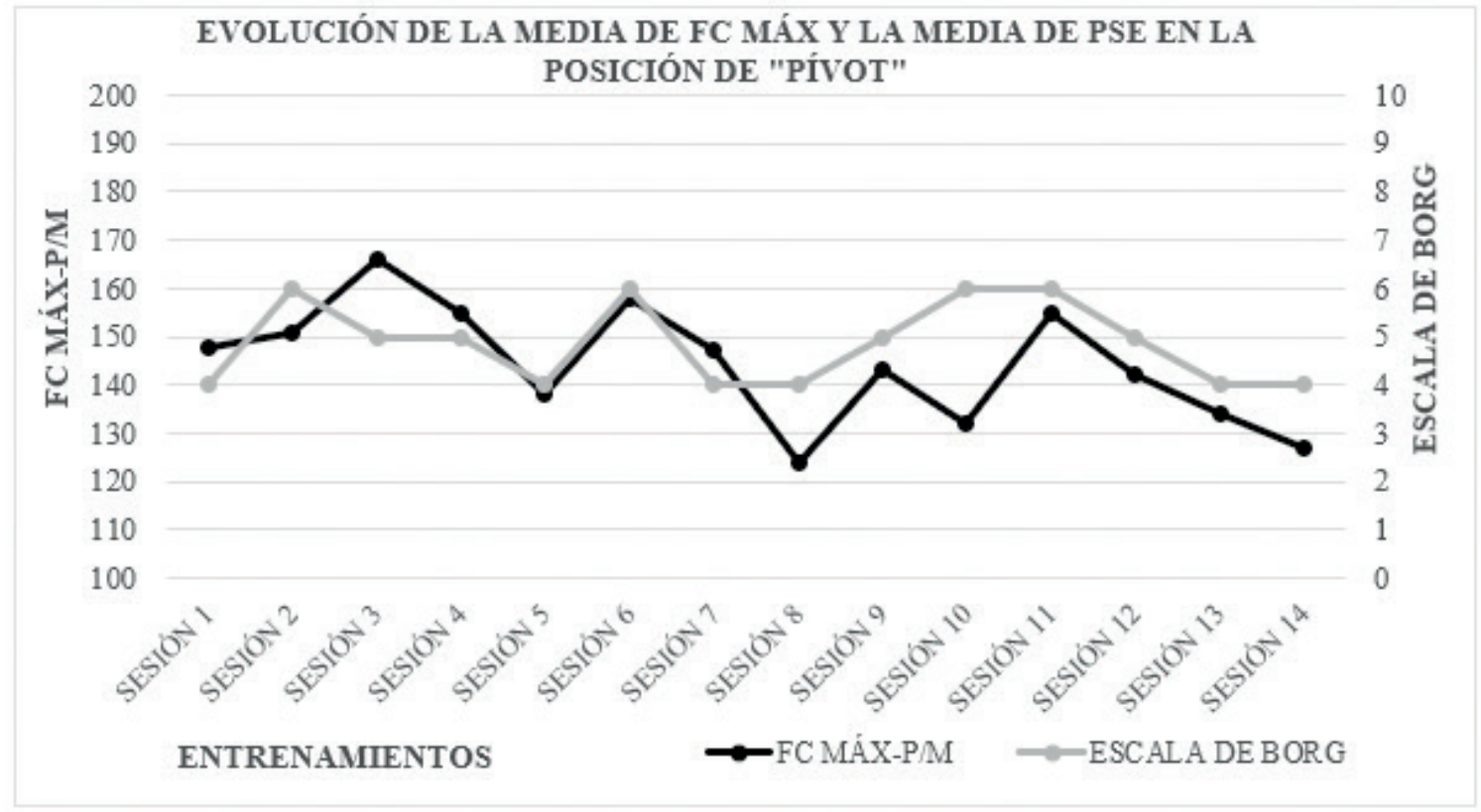

Figura 5. Evolución de la media de FC Máx y la media de PSE en la posición de "Pívot" a los largo de las catorce sesiones de entrenamiento realizadas por los jugadores del Plásticos Romero Cartagena Fútbol Sala.

En la Figura 5, se puede ver una evolución inversa en algunos tramos de la gráfica, y directa entre otros respecto a las variables reflejadas de la media de FC Máx y la media de la PSE en la posición de "Pívot".

Como dato significativo, se puede destacar le sesión 10, en la cual la media de FC Máx había disminuido respecto a la sesión anterior pero por el contrario la media de la PSE había aumentado.

En cuanto a las sesiones finales registradas 12-13-14, se puede ver una relación directa, ya que a medida que se reduce la media de FC Máx, la media de la PSE va disminuyendo.

Se puede destacar que en la posición de "Pívot" se obtuvo una evolución de la media de FC Máx y la media de la PSE con mejor relación directa y una mejor evolución paralela que en el resto de posiciones ("Cierre" y "Ala"). Esto puede deberse a que los jugadores que ocupaban la posición de "Pívot" eran los más veteranos y por tanto podrían tener interiorizado una mejor percepción subjetiva del esfuerzo que los jugadores más jóvenes.

\section{Discusión}

El objetivo de este estudio fue analizar la frecuencia cardiaca máxima y la percepción subjetiva del esfuerzo en jugadores profesionales pertenecientes a un equipo de la primera división de la Liga Nacional de Fútbol Sala (LNFS) en función de la posición ocupada en el terreno de juego ("Cierre", "Ala", "Pívot), y de este modo ver si existen diferencias significativas entre las distintas posiciones y a la vez establecer si existe una relación válida entre la FC y la PSE para cuantificar la carga interna.

Por otro lado determinar si a través del registro de frecuencia cardiaca los valores se acercaban a los registrados en competición por otros estudios.

Hay estudios como el de Cuadrado-Reyes et al., (2012), Calahorro et al., (2013), o el de Murillo, Álvarez y Manonelles (2016), en los que se examina y se corrobora la percepción subjetiva del esfuerzo como método útil a la hora de determinar la fatiga y el esfuerzo en deportes colectivos. Es por esto que después de examinar los resultados recogidos a lo largo de las catorce sesiones de entrenamiento se observa que aun- 
que en la evolución de los entrenamientos haya determinados tramos con relación indirecta entre las medias de FC Máx y las medias de la PSE, se puede decir que puede existir una relación válida entre la frecuencia cardiaca y la percepción subjetiva del esfuerzo en nuestro estudio.

Quizás esto se pueda deber a que en la mayoría de los tramos de evolución a lo largo de las catorce sesiones de entrenamiento existe una relación directa o una evolución paralela entre la frecuencia cardiaca y la percepción subjetiva del esfuerzo.

Además todos los valores recogidos de la media de FC Máx de forma global en el total de las catorce sesiones de entrenamiento han sido muy similares o casi iguales en las tres posiciones de los jugadores ("Cierre", "Ala" y "Pívot") y lo mismo ha ocurrido con la percepción subjetiva del esfuerzo analizada, ya que la media de la PSE de forma global en el total de las catorce sesiones de entrenamiento ha sido igual en las tres posiciones ("Cierre", "Ala” y "Pívot"), por lo que es posible que no haya habido una mala percepción del esfuerzo por parte de los jugadores de ninguna posición.

De esta forma, tanto a través de la frecuencia cardiaca, como a través de la percepción subjetiva del esfuerzo creemos que hemos conseguido unos valores muy parecidos, por lo que la PSE se muestra como un método valido para cuantificar la carga interna.

Seguidamente en relación el análisis de la carga interna y ver cómo afecta la intensidad del entrenamiento en Fútbol Sala en función de la posición que se puede ocupar en el terreno de juego, los resultados obtenidos en este estudio no se han podido comparar con otros estudios similares debido a la inexistencia de investigaciones.

Existen estudios como el de Gómez, San Román-Quintana, Paulis y Calleja-Gonzales (2012), donde se recogieron valores fisiológicos de jugadores no profesionales en partidos de Fútbol 7 en función de la posición que ocupaban, defensa central, medio-banda, medio-centro y delantero, u otros estudios como en el de Jiménez-García, Lucas-Yuste y Pellicer (2010), en el que se realizó una investigación sobre la cantidad de líquido ingerido y el nivel de deshidratación en porteros, defensores y atacantes de fútbol sala durante la competición en tres partidos oficiales.

En nuestro estudio se puede observar, una pequeña diferencia respecto a la carga interna manifestada de forma más exigente y con valores un poco más elevados de frecuencia cardiaca por parte de los jugadores de la posición de "Ala" y "Pívot" respecto a la posición de "Cierre" en la evolución de las catorce sesiones de entrenamiento. Ocurre una circunstancia parecida en cuanto a la evolución de la carga interna establecida en función de la PSE según la escala de Borg a lo largo de las catorce sesiones de entrenamiento, ya que la carga interna manifestada a través de la PSE ha sido más exigente y en un mayor número de sesiones por parte de los jugadores de la posición de "Ala", respecto a la posición de "Pívot" y "Cierre".

Así, si nos centramos en los parámetros fisiológicos de frecuencia cardiaca recogidos de forma global en el total de las catorce sesiones de entrenamiento, se observa que la carga interna también ha sido un poco más intensa y ha supuesto una mayor fatiga a los jugadores que juegan en la posición de "Ala" y "Pívot", ya que se ha obtenido una media de FC Máx de $145 \mathrm{P} / \mathrm{M}$, con una diferencia de $6 \mathrm{P} / \mathrm{M}$ respecto a la posición de "Cierre", en la cual se obtuvo una media de 139 P/M. Por lo que respecta a los datos recogidos en la investigación sobre la PSE en la escala de Borg de forma global en el total de las catorce sesiones de entrenamiento, se consiguió el mismo valor para las tres posiciones, un valor de cinco (5) sobre diez (10) en la escala de Borg, el cual representa la percepción del esfuerzo con la calificación de "Duro".

Por lo tanto no se han conseguido diferencias muy significativas entre la carga interna analizada en función de las tres posiciones ocupadas en el terreno de juego por los jugadores del Plásticos Romero Cartagena Fútbol Sala, ya que aunque en la mayoría de sesiones la media de FC Máx y la media de la PSE hayan sido mayor en la posición de "Ala" y "Pívot", al final, en las medias registradas de forma global en el total de las catorce sesiones de entrenamiento sólo existe una leve diferencia de $6 \mathrm{P} / \mathrm{M}$ en la frecuencia cardiaca.

Esta diferencia no se observa en la PSE, debido a que son valores y parámetros mucho más globales del esfuerzo que la frecuencia cardiaca. Por eso la media global de la PSE en el total de las catorce sesiones de entrenamiento ha sido igual en las tres posiciones ("Cierre", "Ala" y "Pívot").

Teniendo en cuenta estos datos, el deber del preparador físico estaría estrechamente relacionado con analizar esta carga interna y asignar y modificar la carga adecuada en la sesión de entrenamiento para intentar que los jugadores continúen obteniendo adaptaciones y lleguen a un estado de forma física adecuado (Álvarez et al., 2004), y rehuir los problemas de sobresfuerzo o desentrenamiento que una mala planificación de las cargas puede propiciar (Campos-Vázquez, 2012).

Por último si comparamos las medias de FC Máx de los jugadores del Plásticos Romero Cartagena Fútbol Sala en función de la posición ocupada, con relación a las medias de FC Máx en competición según diferentes estudios, se observa que si se analizan los datos recogidos en las investigaciones de Álvarez, Giménez, Corona y Manonelles (2002), en el cual obtuvieron una media de FC Máx de $165 \pm 10 \mathrm{P} / \mathrm{M}$, al igual que en la investigación de Barbero et al., (2004), donde se consiguió una media de FC Máx de $192 \pm 0,8$ P/M en competición.

Y por último los datos establecidos en el estudio de Álvarez et al. (2001), en el cual se determinó una media de FC Máx de $166 \pm 11 \mathrm{P} / \mathrm{M}$, mostramos que los valores recogidos en las sesiones de entrenamiento de nuestra investigación están mayormente por debajo de los datos recogidos en competición por otros estudios. 
De esta forma, los valores de la media de FC Máx de la posición de "Cierre" se alejan de este valor medio en competición, ya que en las catorce sesiones de entrenamiento las medias de FC Máx van desde las 114 hasta las $174 \mathrm{P} / \mathrm{M}$, por lo que en el $92 \%$ de las sesiones la frecuencia cardiaca ha estado por debajo del valor de competición.

El mismo suceso ocurre con los valores de la media de FC Máx de la posición de "Ala", ya que los datos oscilan entre las 132 y 164 P/M en las catorce sesiones de entrenamiento, estando por lo tanto la frecuencia cardiaca el $100 \%$ de las veces por debajo de las medias de frecuencia cardiaca recogidas en competición.

Respecto a la posición de "Pívot" vuelve a ocurrir la misma circunstancia, las medias de FC Máx van desde las 124 hasta las $166 \mathrm{P} / \mathrm{M}$ en las catorce sesiones de entrenamiento, quedando por lo tanto en el $92 \%$ de las veces por debajo de los parámetros de competición.

Como muestran nuestras tablas, los valores obtenidos en nuestra investigación en las catorce sesiones de entrenamiento no coinciden y están muy lejos de los datos y registros de frecuencia cardiaca recogidos en competición por otros estudios.

Por lo tanto, nos hemos encontrado con limitaciones para analizar de forma objetiva nuestro estudio, ya que debería haberse tenido en cuenta la forma en la que se ha recogido y analizado la frecuencia cardiaca en nuestra investigación, debido a que se han recogido datos y valores de todas las partes del entrenamiento, y se ha realizado una media de forma global.

Por eso hay también que destacar que, algunas partes del entrenamiento no son tan exigentes como la competición en sí, por ejemplo el momento del calentamiento.

Por este motivo es complicado establecer una relación y comparar nuestros datos con los registrados en otros estudios e investigaciones en los que sólo se han centrado puramente en la competición, lógicamente esto ha sido una limitación.

Como mejora de la investigación, hubiera resultado más determinante realizar un estudio que hubiera recogido datos durante un periodo más amplio de tiempo, con mayor instrumental científico para medir la frecuencia cardiaca y que todos los jugadores llevasen incorporado un pulsómetro.

Hubiera sido muy interesante haber contado con un medio más avanzado y moderno que nos hubiera facilitado anotar y recoger la frecuencia cardiaca de una forma más sencilla y precisa, que en nuestro estudio ha sido imposible conseguir.

\section{Conclusión}

Después de haber analizado los resultados obtenidos, las conclusiones que establecemos en nuestra investigación en relación a los objetivos marcados serían:
1. Se puede afirmar que existe una relación válida entre la frecuencia cardiaca y la percepción subjetiva del esfuerzo en nuestro estudio en relación a los valores recogidos en la posición de "Cierre", "Ala" y "Pívot". Por lo tanto la PSE se muestra como un método adecuado y como herramienta válida para determinar el esfuerzo establecido por la carga interna en el jugador. De este modo, para clubes con escasos recursos económicos es una herramienta muy útil y de fácil utilización y compresión válida para evaluar el estado de los deportistas, tanto en deportes colectivos como en deportes individuales.

2. Respecto a cómo afecta la intensidad del entrenamiento en función de las diferentes posiciones que pueden ocupar los jugadores en el terreno de juego, no se han conseguido diferencias muy significativas, pero se puede destacar una leve distinción, en la cual los jugadores que juegan en la posición de "Ala" y "Pívot" han desarrollado un esfuerzo mayor y han tenido un mayor gasto energético según el parámetro fisiológico analizado de frecuencia cardiaca.

3. En cuanto a la cercanía de los valores medios de FC Máx de las distintas posiciones analizados en las catorce sesiones de entrenamiento en relación a los recogidos en competición, cabe destacar que se encuentran muy lejos de ellos, y mayoritariamente ninguna posición ("Cierre", "Ala" y "Pívot") se acerca a esas medias de FC Máx en competición real registradas por otras investigaciones.

\section{Futuras líneas de investigación}

Para futuras líneas de investigación sería necesario realizar estudios que se centrasen de manera más profunda en determinar si hay o no, diferencias significativas entre la carga interna de los jugadores de Fútbol Sala en función de la posición que pueden ocupar en el terreno de juego, ya que estudios e investigaciones anteriores se han centrado en cuantificar la intensidad y carga interna de jugadores de Fútbol Sala en competición y en entrenamientos, pero no se ha indagado en mostrar datos relevantes en función de las diferentes posiciones que pueden desempeñar de "Cierre", "Ala" y "Pívot".

Sería necesario conocer el esfuerzo realizado por nuestros diferentes jugadores de campo para la planificación de las siguientes sesiones, y de este modo variar la intensidad e intentar establecer un entrenamiento óptimo para todos los jugadores en función de su posición. De esta forma conseguiremos una mayor eficiencia para todos los jugadores en los entrenamientos y una planificación óptima para alcanzar los objetivos de nuestro equipo. 


\section{Referencias}

1. Álvarez, J., Giménez, L., Manonelles, P., y Corona, P. (2001). Importancia del $\mathrm{VO}_{2}$ Máx y de la capacidad de recuperación en los deportes de prestación mixta. Caso práctico: Fútbol-sala. Archivos de Medicina del Deporte, 18(86), 577-583.

2. Álvarez, J., Giménez, L., Corona, P., y Manonelles, P. (2002). Necesidades cardiovasculares y metabólicas del Fútbol Sala: análisis de la competición. Apunts Educación Física y Deportes, 67 (1), 45-51.

3. Álvarez, J., Manonelles, P., y Corona, P. (2004). Planificación y cuantificación del entrenamiento en una temporada regular de Fútbol Sala. Apunts Educación Fisica y Deporte, 76, 48-52.

4. Álvarez, J., Manonelles, P., Giménez, L., y Nuviala, A. (2009). Incidencia lesional y su repercusión en la planificación del entrenamiento en Fútbol Sala. Archivos de Medicina del Deporte, 26(132), 261-272.

5. Álvarez, J., Murillo, V., Usan, P., Ros, R., y Manonelles, P. (2016). Percepción subjetiva del esfuerzo como método de control de la fatiga y la intensidad en Fútbol Sala. Retos, 30, 9-14.

6. Álvarez, J., y Murillo, V. (2016). Comparación entre las cargas planificadas y ejecutadas en el entrenamiento de fútbol sala: la doble escala. Retos, 29, 48-52.

7. Barbero, J.C., Granda, J., y Soto-Hermoso, V.M. (2004). Análisis de la frecuencia cardiaca durante la competición en jugadores profesionales de fútbol sala. Apunts de Educación Física y Deportes, 77, 71-78.

8. Borg, G. (1990). Psychophysical scaling with applications in physical work and the perception of exertion. Scand J Work Environ Health, 16(1), 55-58.

9. Bortoli de, R., Bortoli de, A.L., y Márquez, S. (2002). Estudio de las capacidades cognoscitivas en el Fútbol Sala. Revista de Psicología del Deporte, 11(1), 53-67.

10. Calahorro, F., Torres-Luque, G., y Lara-Sánchez, A.J. (2013). La percepción subjetiva de esfuerzo como herramienta válida para la monitorización de la intensidad del esfuerzo en competición en jóvenes futbolistas. Cuadernos de Psicología del Deporte, 14(1), 75-82.

11. Campos-Vázquez, M.A. (2012). Control del entrenamiento en Fútbol: posibilidades de actuación. Revista de entrenamiento deportivo/ J. Sports Traininig, 4, 5-10.

12. Castañer, M., Saüch, G., Camerino, O., Sánchez-Algarra, P., y Anguera, M.T. (2014). Percepción de la intensidad al esfuerzo: Un estudio multi-method en actividad física. Cuadernos de Psicología del Deporte, 15(1), 83-88.

13. Cuadrado-Reyes, J., Chirosa-Ríos, L.J., Chirosa-Ríos, I.J., MartínTamayo, I., y Aguilar-Martínez, D. (2012). La percepción subjetiva del esfuerzo para el control de la carga de entrenamiento en una temporada en un equipo de balonmano. Revista de Psicología del Deporte, 21(2), 331-339.

14. Cuadrado, J., y Grimaldi, M. (2011). Medios para cuantificar la carga interna de entrenamiento en deportes de equipo. La frecuencia cardiaca, el consumo de oxígeno, la concentración de lactato en sangre y la percepción subjetiva del esfuerzo: una revisión. Publice Standard, 1-24.

15. García-Jiménez, J.V., y Yuste, J.L. (2010). Pérdida de peso y deshidratación en atacantes durante partidos oficiales de Fútbol Sala. Revista Andaluza de Medicina del Deporte, 3(2), 52-56.

16. Gómez, D., San Román-Quintana, J., Paulis, J., y Calleja-González, J. (2012). Demandas físicas y fisiológicas en jugadores absolutos no profesionales durante partidos de Fútbol 7: Un estudio de caso. Cultura, Ciencia y Deporte (CCD), 7(20), 115-123.

17. Gómez-Díaz, A.J., Bradley, P., Díaz, A., y Pallares, J. (2013). Percepción subjetiva del esfuerzo en Fútbol profesional: relevancia de los indicadores físicos y psicológicos en el entrenamiento y la competición. Anales de Psicología, 29(3), 656-661.

18. Gómez-Díaz, A.J., Pallarés, J., Díaz, A., y Bradley, P. (2013). Cuantificación de la carga física y psicológica en Fútbol profesional: diferencias según el nivel competitivo y efectos sobre el resultado en competición oficial. Revista de Psicología del Deporte, 22, 463-469.

19. Jiménez, J.V., Lucas, J.L., y Pellicer, J.J. (2010). Ingesta de líquids i deshidratació en jugadors professionals de futbol sala en funció de la posició ocupada al terreny de joc. Apunts Medicina Del'Esport, 45(166), $69-74$.

20. Murillo, V., Álvarez, J., y Manonelles, P. (2016). Control training loads through perceived exertion. Prediction of heart rate. Retos, 30, 82-86.

21. Pascual, N., Llorca, V., Carbonell, J.A., y Pérez-Turpín, J.A. (2016). Análisis de la carga interna en los entrenamientos de Fútbol Sala femenino de $1^{a}$ División Nacional. Sportis. Revista Técnico-Cientifica del Deporte Escolar, Educación Física y Psicomotricidad, 2(2), 254-267.

22. Sanabria, Y.D., y Agudelo, C.A. (2011). Programa de preparación física en velocidad de desplazamiento en el Fútbol Sala. Revista Educación física y deporte, 30(2), 629-635.

23. Tapia-López, A. (2017). Propuesta de control de la carga de entrenamiento y la fatiga en equipos son medios económicos. Revista Española de Educación Física y Deporte, 417, 55-69.

24. Vallés Ortega, C., Fernández-Ozcorta, E. J., \& Fierro Suero, S. (2017). Relación entre la complejidad técnico-táctica en la sesión de entrenamiento y la carga interna en baloncesto femenino. SPORT TK-Revista EuroAmericana de Ciencias del Deporte, 6(1), 163-168. 